\title{
Interesse de vegetarianos ou veganos em adquirir hambúrgueres semelhantes à carne
}

\author{
Vegetarian or vegan interest in purchasing meat-like hamburgers \\ Interés vegetariano o vegano en comprar hamburguesas parecidas a la carne
}

Recebido: 29/06/2021 | Revisado: 05/07/2021 | Aceito: 09/07/2021 | Publicado: 21/07/2021

Cristiana Basso
ORCID: https://orcid.org/0000-0003-2001-0110
Universidade Federal de Santa Maria, Brasil
E-mail: cristianabasso1@ gmail.com
Maristela Lopes Bica Marques
ORCID: https://orcid.org/0000-0001-5652-1217
Universidade Franciscana, Brasil
E-mail: maristela52@ @otmail.com
Rodrigo Fioravanti Pereira
ORCID: https://orcid.org/0000-0003-4129-6568
Universidade Franciscana, Brasil
Neila Richards
ORCID: https://orcid.org/0000-0001-6610-5567
Universidade Federal de Santa Maria, Brasil
E-mail: neilarichardsprof@ gmail.com

\begin{abstract}
Resumo
A opção por se tornar vegetariano ou vegano teve um aumento significativo nos últimos anos, assim, o objetivo deste trabalho foi investigar se o público vegetariano ou vegano possui interesse em adquirir hambúrgueres semelhantes à carne, como os à base de vegetais, ou se ao contrário, estes produtos causam repulsa. Foi aplicado um questionário individual eletrônico, onde solicitou-se aos participantes vegetarianos ou veganos informações pessoais e perguntas relacionadas à sua opção alimentar. Os 136 respondentes foram em sua maioria um público de 18 a 33 anos, do gênero feminino. Percebeu-se que a maioria não apresentou repulsa, inclusive muitos já provaram e gostaram, entendendo como uma alternativa na alimentação, desde que cobrado um preço justo, ou seja, semelhante aos produtos cárneos. As razões citadas pelas quais os participantes adotaram a dieta vegetariana ou vegana foram a maioria em respeito aos animais, seguida pelo respeito ao meio ambiente. Ao se analisar as respostas ficou evidente que não há repulsa por produto semelhante a carne, como hambúrguer a base de vegetais, com isso há uma percepção da busca por variados produtos e pelo interesse em ser uma alternativa na alimentação, com potencial de venda.
\end{abstract}

Palavras-chave: Vegetarianismo; Veganismo; Alternativa; Alimentação.

\begin{abstract}
The option to become vegetarian or vegan has increased significantly in recent years, thus, the objective of this work was to investigate whether the vegetarian or vegan public is interested in purchasing meat-like hamburgers, such as vegetable-based ones, or if on the contrary, these products cause revulsion. An individual electronic questionnaire was applied, in which participants were asked to provide personal information and answer questions related to their choice of food. The 136 respondents were mostly female, in between 18 and 33 years old. It was noticed that the majority did not show disgust, and many have already even tasted and liked it, considering it as an alternative food, as long as fair prices were charged, that is, similar to meat products. The reasons cited as for why participants adopted a vegetarian or vegan diet were mostly in respect for animals, followed by respect for the environment. When analyzing the responses, it was evident that there is no repulsion for a product similar to beef, such as a vegetable-based hamburger and, therefore, there is a perception of the search for varied products and the interest in being an alternative food with potential for sale.
\end{abstract}

Keywords: Vegetarianism, Veganism, Alternative, Food.

\section{Resumen}

La opción de hacerse vegetariano o vegano se há incrementado significativamente en los últimos años, por lo que el objetivo de este trabajo fue investigar si el público vegetariano o vegano está interesado en comprar hamburguesas parecidas a la carne, como las de origen vegetal, o si al contrario, estos productos provocan repulsión. Se aplicó un cuestionario electrónico individual, en el que se pidió a los participantes que proporcionaran información personal y respondieran preguntas relacionadas con su elección de alimentos. Los 136 encuestados eran en su mayoría mujeres, entre 18 y 33 años. Se notó que la mayoría no mostró disgusto, y muchos incluso ya lo probaron y les gustó, considerándolo como un alimento alternativo, siempre y cuando se cobraran precios justos, es decir, similares a los productos cárnicos. Las razones citadas de por qué los participantes adoptaron una dieta vegetariana o vegana fueron 
en su mayoría por respeto a los animales, seguido por respeto al medio ambiente. Al analizar las respuestas, se evidenció que no existe repulsión por un producto similar a la carne, como una hamburguesa a base de vegetales y, por lo tanto, se percibe la búsqueda de productos variados y el interés por ser un alimento alternativo con potencial de venta.

Palabras clave: Vegetarianismo, Veganismo, Alternativa, Comida.

\section{Introdução}

Um nicho de mercado em ascensão tem sido o de alimentos para vegetarianos e veganos, alavancado possivelmente pela preocupação dos consumidores em relação ao impacto da alimentação em sua saúde, ao meio ambiente e ao bem-estar animal (Révillion, Kapp, Badejo \& Dias, 2020).

Com a finalidade de conscientizar as pessoas em suas atividades diárias, inúmeros são os segmentos que tendem a abordar uma vida menos consumista e mais sustentável, como é o caso dos vegetarianos e veganos (Trigueiro, 2013).

O vegetarianismo é dividido em subgrupos, onde cada um deles possui diferentes formas de se alimentar, como os adeptos da dieta Ovolactovegetariana que tem como princípio manter uma dieta sem o consumo de carne de nenhum animal, optando por leite, ovos e seus derivados, que são consumidos normalmente e fornecem todos os nutrientes necessários ao organismo humano; outro subgrupo são os Lactovegetarianos, estes incluem apenas laticínios, deste modo utilizam leite, produtos de origem vegetal, como cereais, verduras, leguminosas, além do mel; e os Ovovegetarianos, que são aqueles que incluem em seu cardápio apenas ovos e vegetais, excluindo os laticínios e carnes. Na dieta vegetariana estrita há uma preocupação com os animais e devido a isso não há o consumo de nenhum tipo de alimento de origem animal, sendo as principais motivações bem parecidas com as dos veganos, porém a restrição se dá apenas a nível alimentar (SVB, 2020).

Merli e Vidigal (2020), expõem uma classificação semelhante, com vegetariano estrito, ovolactovegetariano, lactovegetariano, ovovegetariano, porém acrescentam os pescovegetarianos, que consomem apenas o peixe.

Em relação ao veganismo, os adeptos são aqueles indivíduos que se posicionam contra qualquer modo de exploração animal, seja na alimentação, vestuário, cosméticos, entre outros. Buscam o equilíbrio moral entre animais e humanos. A dieta dos veganos é baseada em vegetais, livre de alimentos de origem animal como carne, laticínios, ovos, mel. Também não utilizam produtos como couro e lã, e os que são testados em animais (Trigueiro, 2013).

Indivíduos que optam por não consumir alimentos de origem animal, certamente sabem que esses alimentos apresentam excelente fonte de proteínas, portanto necessitam adquirir outras fontes proteicas para prover suas necessidades, porém alguns autores relatam que ainda há dificuldade em encontrar produtos diversificados que supram essa necessidade, e que satisfaçam ao paladar, a um custo acessível (Montebello \& Araújo, 2009).

Por outro lado, pesquisadores como Révillion, Kapp, Badejo e Dias (2020) alegam que a demanda de vegetarianos e veganos tem levado a uma crescente oferta de produtos substitutos aos de origem animal, encontrados em lojas especializadas, redes de supermercados, redes de restaurantes e vendas diretas pela internet em sites especializados.

Também Cavalheiro, Verdu e Amarante (2018) sugerem que o movimento vegetariano e vegano tem dado respostas às demandas reprimidas de um público específico, com crescimento a partir da iniciativa de grandes redes de fast-food que passam a incluir opções vegetarianas ou veganas em seus cardápios, ou a partir da abertura de unidades dedicadas exclusivamente a esse segmento.

Corroborando com a forma de pensar de vegetarianos e veganos, principalmente em relação a sustentabilidade, saúde e ética, pesquisa realizada pela Brasil Food Trends 2020 identificou exigências e tendências dos consumidores mundiais de alimentos, agrupando-os em cinco categorias: sensorialidade e prazer; saudabilidade e bem-estar; conveniência e praticidade; confiabilidade e qualidade; sustentabilidade e ética (Federação das Indústrias do Estado de São Paulo FIESP ; Instituto de Tecnologia de Alimentos Ital , 2010). 
Assim a indústria tem buscado desenvolver produtos que satisfaçam essas exigências, sendo possível se deparar em supermercados com produtos vegetarianos e veganos como nuggets, presuntos, quibes, coxinhas, salsichas, linguiças, sorvetes, requeijões, hambúrgueres à base de vegetais (SVB, 2020).

Em vista disso, o presente trabalho se propôs a investigar se o público vegetariano ou vegano possui interesse em adquirir hambúrgueres semelhantes à carne, como os à base de vegetais, ou se ao contrário, estes produtos causam repulsa.

\section{Metodologia}

Tratou-se de um estudo descritivo quantitativo, desenvolvido com participantes que se-autodeclararam vegetariano ou vegano. A coleta dos dados ocorreu de agosto a outubro de 2020 e o protocolo da pesquisa foi aprovado pelo Comitê de Ética e Pesquisa (CEP) da Universidade Franciscana (parecer: número 35089720.8.0000.5306).

Foram incluídos na pesquisa todos os participantes que preencheram os critérios de inclusão, como, ter mais de 18 anos de idade, residir em Santa Maria/RS e concordar com o Termo de Consentimento Livre e Esclarecido (TCLE); e excluídos participantes de outras cidades, menores de 18 anos de idade e os que não concordaram com o TCLE, o qual continha explicações e objetivo da pesquisa.

Foi aplicado aos participantes um questionário individual eletrônico elaborado na plataforma virtual Google Forms, onde eles preenchiam informações pessoais: nome, gênero, idade e e-mail. Posteriormente respondiam perguntas relacionadas ao interesse em consumir hambúrgueres semelhantes a carne.

O questionário foi constituído por 9 perguntas, constando:

- Você se considera ovolactovegetariano; lactovegetariano; ovovegetariano; vegetariano estrito ou vegano?

- Quanto tempo faz que optou em tornar-se vegetariano/vegano? Desde o nascimento; menos de seis meses; de seis meses a um ano; de um a dois anos; mais de dois anos?

- Qual o principal motivo que o levou a se tornar vegetariano/vegano? Não gostava do sabor da carne; em respeito aos animais; motivos religiosos; manter uma alimentação mais leve, mais saudável; em respeito ao meio ambiente; outros?

- $\quad$ Antes de se tornar vegano/vegetariano consumia hambúrguer de carne? Sim ou não; Se SIM, sente vontade de consumir novamente? Sim ou não?

- Você consumiria um hambúrguer com textura, cor e aroma semelhante ao de carne bovina, porém totalmente elaborado com ingredientes a base de vegetais? Sim, somente para experimentar; sim para ser uma alternativa na alimentação; não consumiria?

- Você já provou o hambúrguer a base de vegetais? Sim, mas não gostei; sim, e gostei; não provei.

- Qual seria sua aceitação frente a produtos semelhantes a carne, onde zero significa repulsa total e cinco significa nenhuma repulsa a estes produtos? 0 - repulsa total a estes produtos; 5- nenhuma repulsa a estes produtos; caso haja repulsa, qual seria o motivo?

- $\quad$ Se tratando de custos, para consumir um hambúrguer a base de vegetais, você estaria disposto a pagar um valor inferior ao cobrado por um hambúrguer de carne; um valor igual ao cobrado por um hambúrguer de carne; um valor superior ao cobrado por um hambúrguer de carne?

- $\quad$ Após a conclusão do trabalho deseja receber por e-mail os resultados? Sim ou não?

A divulgação ocorreu por meio das redes sociais e endereços de e-mails, onde foi disponibilizado o link do site com o Termo de Consentimento Livre e Esclarecido e o questionário, assim, o participante era automaticamente direcionado a página específica. 
Os dados foram tabulados no programa Microsoft Excel (versão 2016) e analisados com auxílio do Excel e do software IBM SPSS (versão 25.0), onde passaram por análise exploratória levando em conta as medidas, como média e desvio padrão, para as variáveis contínuas; e frequência absoluta e relativa para as variáveis qualitativas e discretas.

As variáveis foram distribuídas em tabelas de contingência sempre que possível, a fim de investigar a associação entre elas. Havendo os pressupostos cabíveis, o teste Qui-quadrado para associação foi conduzido.

Foram realizados testes estatísticos confirmatórios para identificar diferenças significativas entre as respostas quando separadas por gênero, idade ou tipo declarado de vegetariano ou vegano, entre outras possibilidades apontadas na análise exploratória. Todos os testes estatísticos levaram em conta um nível de significância de 5\%.

\section{Resultados}

A amostra resultou em 136 respostas de indivíduos residentes em Santa Maria/RS, sendo a maioria do sexo feminino (75\%), seguido do sexo masculino (22,8\%) e de outros (2,2\%), todos com idade entre 18 a 61 anos, porém não houve diferença significativa entre as idades quando separadas por gênero (teste de Kruskal Wallis, $\mathrm{p}=0,127$ ), autodeclarados vegetarianos ou veganos (teste de Kruskal Wallis, $\mathrm{p}=0,725$ ).

No que se referiu a classificação, a maior parte dos participantes da pesquisa se intitularam como ovolactovegetarianos $(55,1 \%)$, seguido de vegetarianos estritos (16,2\%), veganos (15,4\%), lactovegetarianos $(10,3 \%)$ e ovovegetarianos (3\%). Referiram também, que aderiram a essa prática a mais de dois anos $(50 \%)$, seguido pelos que relataram de um a dois anos (17,6\%), de seis meses a um ano (17,6\%), menos de seis meses (14\%).

Com relação ao principal motivo que levou o participante a se tornar vegetariano/vegano, $63 \%$ referiram ser por respeito aos animais; seguido de respeito ao meio ambiente (13\%); não gostar do sabor da carne (9\%); para manter uma alimentação mais leve, mais saudável (7\%).

Quanto ao questionamento referente à ingestão de hambúrguer de carne anteriormente a se tornar vegetariano/vegano, 95\% respondeu que consumia, destes, 87,5\% afirmaram não sentir vontade de consumir novamente.

Ao serem questionados se consumiriam um hambúrguer semelhante ao de carne bovina, mas elaborado à base de vegetais, as respostas evidenciaram que a maioria consumiria por ser uma alternativa na alimentação $(62,5 \%)$, outros somente para experimentar $(22,1 \%)$, e a minoria referiu que não consumiria $(15,4 \%)$.

Em relação a já terem provado hambúrguer à base de vegetais, 72,8\% dos participantes provaram e gostaram; 16,9\% não provaram e 10,3\% provaram, mas não gostaram. No quadro 1, são apresentados o percentual das variáveis ou características referentes a aceitação de hambúrguer semelhante a carne e o preço que estaria disposto a pagar. 
Quadro 1. Resultado das variáveis relacionadas a vegetarianos e veganos residentes em Santa Maria, Rio Grande do Sul, 2020.

\begin{tabular}{|l|c|}
\hline Variável ou Característica & Porcentagem (\%) \\
\hline Qual seria sua aceitação frente a produtos semelhantes a carne, onde zero significa \\
repulsa total e cinco significa nenhuma repulsa a estes produtos & 11,8 \\
0, repulsa total a estes produtos & 4,4 \\
1, & 4,4 \\
2, & 18,4 \\
3, & 7,4 \\
4, & 53,6 \\
5, nenhuma repulsa a estes produtos & \\
\hline Quanto estaria disposto a pagar (R\$) por um hambúrguer à base de vegetais & 25,8 \\
Um valor inferior ao cobrado por um hambúrguer de carne & 56,6 \\
Um valor igual ao cobrado por um hambúrguer de carne & 17,6 \\
Um valor superior ao cobrado por um hambúrguer de carne & \\
\hline
\end{tabular}

Fonte: Autores.

Em relação a possível resistência dos vegetarianos e veganos em tolerar hambúrgueres semelhantes a carne bovina, percebeu-se que a maioria dos participantes não apresentou repulsa (Quadro 2).

Quadro 2. Frequências de respostas ao questionário com o nível de repulsa dos participantes veganos e vegetarianos, Santa Maria, Rio Grande do Sul, 2020.

\begin{tabular}{|c|c|c|c|c|c|c|}
\hline \multirow{2}{*}{ Variável ou Característica } & \multicolumn{6}{|c|}{ Nível de Repulsa } \\
\hline & $\mathbf{0}$ & $\mathbf{1}$ & 2 & 3 & 4 & 5 \\
\hline \multicolumn{7}{|l|}{ Você se considera (n) } \\
\hline Lactovegetariano & 1 & 0 & 0 & 0 & 0 & 13 \\
\hline Ovolactovegetariano & 7 & 3 & 3 & 17 & 9 & 36 \\
\hline Ovovegetariano & 0 & 1 & 0 & 1 & 0 & 2 \\
\hline Vegetariano Estrito & 3 & 0 & 1 & 3 & 0 & 15 \\
\hline Vegano & 5 & 2 & 2 & 4 & 1 & 7 \\
\hline \multicolumn{7}{|l|}{ Tempo de opção (n) } \\
\hline Desde o nascimento & 0 & 1 & 0 & 0 & 0 & 0 \\
\hline Menos de seis meses & 1 & 1 & 1 & 4 & 1 & 11 \\
\hline De seis meses a um ano & 2 & 1 & 1 & 3 & 3 & 14 \\
\hline De um a dois anos & 4 & 0 & 1 & 1 & 3 & 15 \\
\hline Mais de dois anos & 9 & 3 & 3 & 17 & 3 & 33 \\
\hline
\end{tabular}




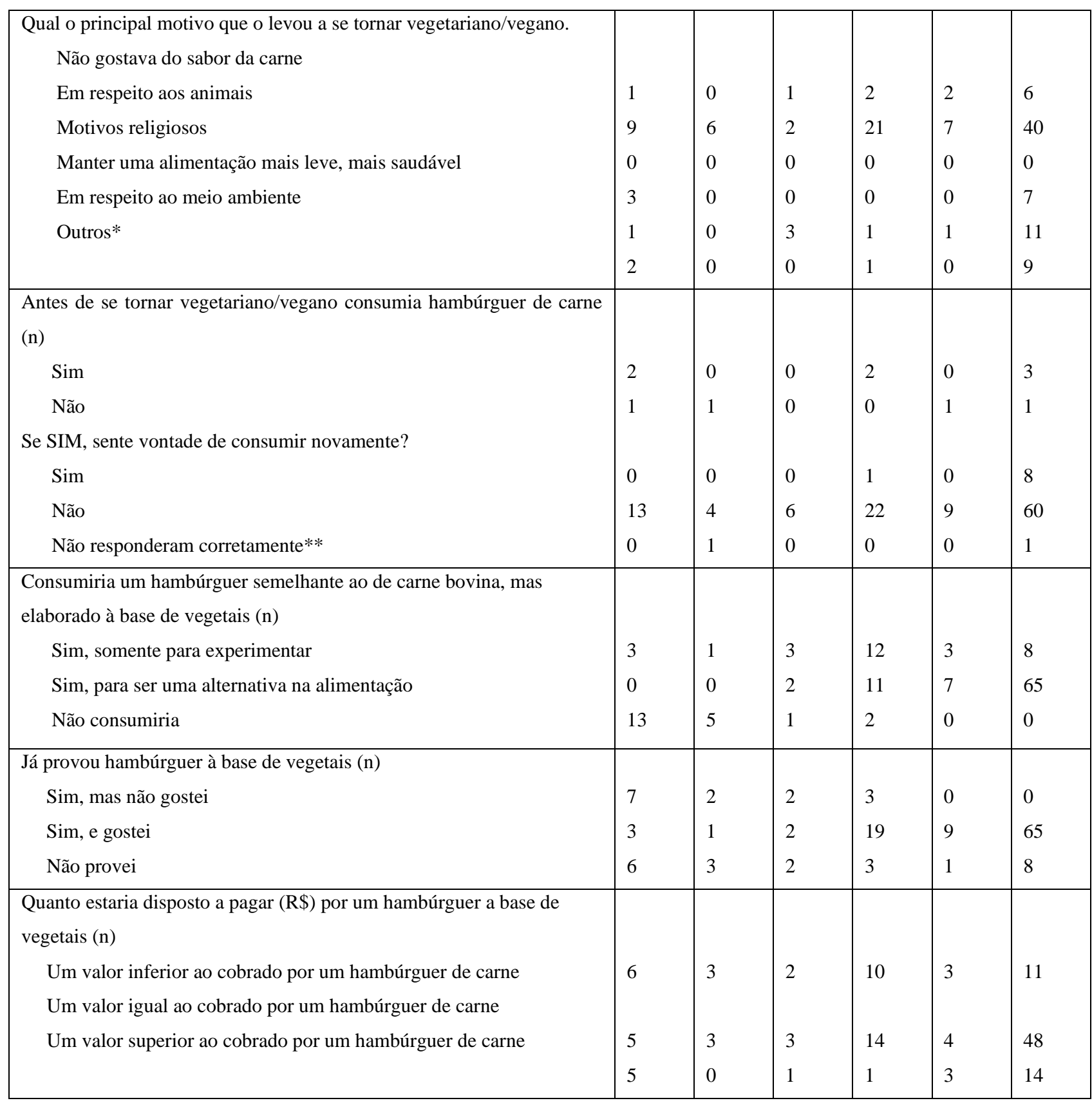

Zero significa repulsa total e cinco indica nenhuma repulsa.

*Indica que houve pessoas que responderam mais de uma opção, sendo considerado como resposta livre.

**Não responderam corretamente, pois era para responder somente quem já consumia hambúrguer de carne.

Fonte: construção dos autores

\section{Discussão}

Segundos últimos dados do Instituto Brasileiro de Opinião Pública e Estatística (IBOPE, 2018), o percentual da população que se autodeclara vegetariana no Brasil atingiu 14\%, equivalendo a 29,2 milhões de pessoas no ano de 2018, conferindo um aumento significativo de 75\% em relação ao ano de 2012, já quanto aos adeptos do veganismo, ainda não há registros mais específicos (SVB, 2020).

De acordo com IBOPE (2018), a frequência entre homens e mulheres vegetarianos no Brasil é semelhante, diferente dos resultados encontrados no presente estudo, em que o montante de mulheres predominou. Também Ruby (2012) constatou que em muitos países ocidentais a quantidade de mulheres vegetarianas é maior que a de homens, além de Barros, Bierhals e 
Assunção (2020) que ao pesquisar o vegetarianismo entre ingressantes de uma universidade pública no sul do Brasil associaram a referida dieta ao gênero feminino $(54,8 \%)$ e ao uso de álcool.

Também Shen, Chang, Lin e Lin (2021) comprovaram que a dieta vegetariana estaria associada com menor risco de depressão em Taiwan, e que a maioria dos participantes vegetarianos eram do gênero feminino (74\%). Além de Carvalho e Moreira (2020) que da mesma forma perceberam em estudo realizado com vegetarianos não estritos e vegetarianos estritos e/ou veganos que a amostra foi composta em sua maioria por mulheres (79,3\% vegetarianas não estritas e $82 \%$ vegetarianas estritas).

O estudo de Queiroz, Soliguetti e Moretti (2018) apontou que o público vegetariano e vegano é bastante jovem, 53\% entre 18 a 25 anos, $30 \%$ de 26 e 35 anos,corroborando com o resultado encontrado nessa pesquisa onde encontrou-se as faixas etárias mais predominante de 18 anos a 33 anos. Também na pesquisa realizada por Barros et al. (2020) predominou indivíduos mais jovens adeptos ao vegetarianismo, contanto com idade entre 18 a 19 anos em 41,2\%, seguido de idade entre 20 a 22 anos em $32,8 \%$.

Por vezes, no entanto, percebe-se algumas atitudes impróprias cometidas por esse público, como por exemplo, sabe-se que a idade com que os indivíduos começam a se tornar vegetarianos desempenha um papel importante no desenvolvimento de comportamentos extremos e não saudáveis, como no controle de peso, tendo por base algumas pesquisas que sugerem uma associação entre o vegetarianismo e os distúrbios alimentares (Timko, Hormes \& Chubski, 2012), além da observação feita por Barros et al. (2020) em relação a associação com o consumo de álcool.

Outro agravante, é que embora a alimentação vegetariana e vegana esteja frequentemente relacionada a mais saudável, estudo realizado por Silveira, Meneses, Quintana e Santos (2017) identificou frequência elevada do consumo diário de bebidas açucaradas e alimentos ultraprocessados, associado ao excesso de peso.

De forma geral, a escolha por uma dieta vegetariana pode levar a resultados positivos, como melhor saúde física, ser moralmente correta e gerar menor impacto ambiental, por outro lado, pode ter um impacto negativo na qualidade de vida de quem opta por se abster de carnes ou outros produtos de origem animal, levando a deficiências nutricionais e dependendo da questão econômica a um acesso limitado a uma ampla variedade de alimentos vegetais (Hargreaves, Raposo, Saraiva \& Zandonadi, 2021).

No que diz respeito aos subgrupos, observou-se no presente estudo a prevalência de ovolactovegetarianos $(55,1 \%)$. Conforme Slywitch (2012), estudo realizado com 664 indivíduos vegetarianos avaliados na cidade de São Paulo, demonstrou que $67 \%$ eram ovolactovegetarianos, $22 \%$ eram vegetarianos estrito, $10 \%$ eram lactovegetarianos, $8 \%$ eram veganos e $1 \%$ era ovovegetariano.

Apesar de haver um crescimento nos grupos de vegetarianos, esse número na população brasileira ainda é pequeno, portanto, o consumo de alimentos de origem animal como ovos, leite e derivados ainda é alto (Weber, Fraga, Alcântara \& Setaro, 2017), justificando provavelmente o fato de que predomina o grupo dos ovolactovegetarianos, que por ser mais flexível torna-se a mais popular entre os subgrupos dos vegetarianos.

Diante dos resultados obtidos percebeu-se com relação ao tempo de opção que metade dos participantes adotaram esse estilo de vida a mais de dois anos. Corroborando, Silveira et al. (2017), obtiveram em seu estudo um tempo mediano de vegetarianismo de 5,3 anos, além de evidenciar na amostra de 503 indivíduos, 83,7\% de mulheres e os tipos mais frequentes de vegetarianismo foram ovolactovegetarianos $(45,4 \%)$, seguido de veganos $(41,7 \%)$.

Já Carvalho e Moreira (2020) constataram que a amostra, composta por 198 não estritos e 200 estritos, com médias de idade de 28,5 anos e 31,5 anos respectivamente, adotava esse estilo de vida atual em sua maioria há 3 anos (58,1\%) e pouco menos a mais de 3 anos (41,9\%), sendo predominante também entre os vegetarianos não estritos a dieta ovolactovegetariana (77,3\%). 
As razões pelas quais os participantes adotaram a dieta vegetariana ou vegana foram: a maioria em respeito aos animais, seguida em respeito ao meio ambiente, não gostava do sabor da carne, para manter uma alimentação mais leve e por outros motivos, os quais foram citados pelos participantes sendo eles: as condições precárias nos frigoríficos, decisão política, problemas com digestão, impacto causado pelo consumo da carne. Corroborando, Queiroz, Soliguetti \& Moretti (2018), observaram que entre 214 pessoas que seguiam alguma dieta vegetariana, 53\% apontaram o respeito aos direitos dos animais como o motivador mais significativo para escolha de tal hábito alimentar.

Assim, conforme Schinaider (2018), o principal motivo de adesão não é a saúde, mas sim a ética, devido os adeptos defenderem os direitos dos animais e afirmarem ser injusto e cruel a matança e o sofrimento do animal para o consumo humano em função dos processos envolvidos. Resultado que vem ao encontro dessa pesquisa em que a maioria dos participantes relataram não sentir vontade de consumir hambúrguer novamente, embora a maioria não sinta nenhuma repulsa em ingerir produto semelhante a carne.

Percebe-se então que a maioria dos adeptos faz a escolha por motivo ético, com base na ideia de que o abate de animais para consumo humano é moralmente impróprio. Outra motivação importante é a saúde e os potenciais efeitos benéficos do vegetarianismo, além da preocupação com os impactos ambientais da produção de carne (Hargreaves, Raposo, Saraiva \& Zandonadi, 2021).

O fato é que a alimentação vegetariana e vegana causa menos poluição e consome menos recursos para ser produzida, em comparação a uma alimentação com produtos animais. Desse modo, a indústria vem buscando desenvolver produtos inovadores para suprir as necessidades deste público, como o hambúrguer à base de vegetais, por exemplo, sendo mais uma alternativa na tentativa de diversificar a alimentação (SVB, 2020).

Os resultados também mostraram que a maioria dos participantes consumiam hambúrguer de carne bovina antes de se tornarem vegetarianos ou veganos, e isso indica que mais da metade da amostra acredita que hambúrgueres elaborados a base de plantas seria uma boa alternativa para a alimentação.

Assim, com essa demanda crescente, mais pesquisas são realizadas, como a de Lima (2008), em que elaborou hambúrguer vegetal à base de caju para caracterização físico-química e sensorial, em comparação com hambúrguer de carne bovina, de proteína de soja, de proteína vegetal e de proteína vegetal sabor frango, resultando em uma alternativa promissora.

De acordo com a Sociedade Vegetariana Brasileira (SVB, 2020), a quantidade de carne que será consumida até o ano de 2040 não virá de animais abatidos, devendo ser mais de 50\% cultivada em laboratórios ou provinda de produtos à base de vegetais, com a mesma textura, aparência, cor e sabor da carne bovina. Nota-se, portanto, que dentro da amostra estudada, a maioria dos participantes não sentiriam nenhuma repulsa a estes produtos semelhantes a carne, tanto que $72,8 \%$ já provaram e gostaram do hambúrguer à base de vegetais, criando uma tendência de venda de mercado.

A partir do resultado obtido em relação a custo, a maioria dos participantes estaria disposta a pagar um valor igual ao cobrado por hambúrguer de carne. Percebe-se que os consumidores escolhem produtos com base nos preços e nas características sensoriais como sabor, aroma, textura e cor do alimento, que deverão ser agradáveis ao paladar do público. Ressaltando, pesquisa de Brandel, Penha, Sari e Hackenhaar (2019) sobre o grau de importância de diferentes fatores na compra de alimentos, constatou que a maioria dos entrevistados consideram o sabor do alimento na hora da compra, seguido da qualidade e preço do produto.

\section{Conclusão}

Ao analisar as respostas dos participantes, fica evidente que não há repulsa por produtos semelhantes a carne bovina, inclusive podendo ser mais uma alternativa na alimentação. Sendo assim, é possível destacar que o hambúrguer a base de vegetais parece ter um grande potencial de venda, quando cobrado um valor semelhante ao preparado com carne. 
O mercado de alimentos para os consumidores vegetarianos e veganos parece estar em forte crescimento e ainda existe muito o que explorar no desenvolvimento de novos produtos, que podem ser oportunidades para empresas, que agradarão a esse público, além de não atentar contra os animais, a saúde, o impacto ambiental, contexto social e econômico.

\section{Referências}

Barros, K. S., Bierhals, I. O., \& Assunção, M. C. F. (2020). Vegetarianismo entre ingressantes de uma universidade pública no sul do Brasil, 2018. Epidemiologia e serviços de saúde, 29(4), 1-11.

Brandel, G. T, Penha, J. L. J, Sari, L. P \& Hackenhaar, M. L. (2019). Grau de importância na compra de produtos alimentícios em relação à qualidade, sabor, preço, marca e embalagem. In V Mostra de Trabalhos do Curso de Nutrição do Univag.

Carvalho, R. C. de, \& Moreira, J. M. (2020). Níveis de concordância com regras descritivas sobre comportamentos alimentares entre veg(etari)anos. Psico$U S F, 25(3), 533-545$.

Cavalheiro, C. A., Verdu, F. C. \& Amarante, J. M. (2018). Difusão do vegetarianismo e veganismo no brasil a partir de uma perspectiva de transnacionalização. Revista eletrônica ciências da administração e turismo, 6(1), 51-67.

Federação das Indústrias do Estado de São Paulo (FIESP); Instituto de Tecnologia de Alimentos (Ital). (2010). Brasil Food Trends 2020. 176p.

Hargreaves, S. M., Raposo, A., Saraiva, A., \& Zandonadi, R. P. (2021). Dieta vegetariana: uma visão geral através da perspectiva dos domínios de qualidade de vida. International Journal of Environmental Research and Public Health, 18, 1- 23.

Ibope (2018). 14\% da população se declara vegetariana. http://www.ibopeinteligencia.com/noticias-e-pesquisas/14-da-populacao-se-declara-vegetariana/

Lima, J. R. (2008). Caracterização físico-química e sensorial de hambúrguer vegetal elaborado à base de caju. Ciência e agrotecnologia, $32(1)$, $191-195$.

Merli, I. J., \& Vidigal, F. de C. (2020). Perfil antropométrico, dietético e pressão arterial de adultos onívoros e vegetarianos. Nutrición clinica y dietética hospitalaria, 40(3), 60-68.

Montebello, N. P.; \& Araújo, W. M. C. (2009). Carne \& Cia - Série Alimentos e Bebidas. Senac. 324 p.

Queiroz, C. A., Soliguetti, D. F. G., \& Moretti, S. L. do A. (2018). As principais dificuldades para vegetarianos se tornarem veganos: um estudo com o consumidor brasileiro. Demetra, 13(3), 535-554.

Révillion, J. P., Kapp, C. Badejo, M. S. \& Dias, V da V. (2020). O mercado de alimentos vegetarianos e veganos: características e perspectivas. Cadernos de Ciência \& Tecnologia, 37(1), 1-10.

Ruby, M. B. (2012). Vegetarianism. A blossoming field of study. Appetite, 58(1), 141-150.

Shen, Y., C., Chang, C., E., Lin, M. N., \& Lin, C. L. (2021). Vegetarian Diet Is Associated with Lower Risk of Depression in Taiwan. Nutrients, 13, 1-13.

Silveira, J. A. C. da, Meneses, S. S., Quintana, P. T., \& Santos. (2017). Association between overweight and consumption of ultra-processed food and sugarsweetened beverages among vegetarians. Revista de Nutrição, 30(4), 431- 441.

Slywicth, E. (2012). Guia alimentar de dietas vegetarianas para adultos. Departamento de Medicina e Nutrição, Sociedade Vegetariana Brasileira. 66p.

Sociedade Vegetariana Brasileira. (2020). Vegetarianismo: o que é. São Paulo, https://bit.ly/3qu5tks

Timko, C., Hormes, J. M., \& Chubski, J. (2012). Will the real vegetarian please stand up? An investigation of dietary restraint and eating disorder symptoms in vegetarians versus non-vegetarians. Appetite, 58(3), 982-990.

Trigueiro A. (2013). Consumo, ética e natureza: o veganismo e as interfaces de uma política de vida. Revista Internacional Interdisciplinar INTERthesis, $10(1), 237-260$.

Weber, M. L., Fraga, K. S., Alcântara, P. H. G. de, \& Setaro, L. (2017). Adequação do consumo alimentar de adeptos da dieta vegetariana estrita. LifeStyle Journal, 4(2):73-86. 